\title{
Medial auditory thalamus is necessary for acquisition and retention of eyeblink conditioning to cochlear nucleus stimulation
}

\author{
Hunter E. Halverson, Amy Poremba, and John H. Freeman \\ Department of Psychology, University of lowa, lowa City, lowa 52242, USA
}

\begin{abstract}
Associative learning tasks commonly involve an auditory stimulus, which must be projected through the auditory system to the sites of memory induction for learning to occur. The cochlear nucleus $(\mathrm{CN})$ projection to the pontine nuclei has been posited as the necessary auditory pathway for cerebellar learning, including eyeblink conditioning. However, the medial auditory thalamic nuclei (MATN), consisting of the medial division of the medial geniculate, suprageniculate, and posterior interlaminar nucleus have also been implicated as a critical auditory relay to the pontine nuclei for cerebellum-dependent motor learning. The MATN also conveys auditory information to the amygdala necessary for avoidance and fear conditioning. The current study used CN stimulation to increase activity in the pontine nuclei, relative to a tone stimulus, and possibly provide sufficient input to the cerebellum for acquisition or retention of eyeblink conditioning during MATN inactivation. Primary and secondary effects of $\mathrm{CN}$ stimulation and MATN inactivation were examined using 2-deoxyglucose autoradiography. Stimulation of $\mathrm{CN}$ increased activity in the pontine nuclei, however, this increase was not sufficient for cerebellar learning during MATN inactivation. Results of the current experiment provide additional evidence indicating the MATN may be the critical auditory relay for many associative learning tasks.
\end{abstract}

Pavlovian eyeblink conditioning has been widely used to examine the behavioral and neural correlates of associative learning and memory (Gormezano et al. 1983; Christian and Thompson 2003; Freeman and Steinmetz 2011). This task is established by pairing a conditioned stimulus (CS), typically a tone, with an unconditioned stimulus (US), mild periorbital shock, that evokes an eyeblink reflex. After repeated CS-US presentations, an adaptive eyeblink conditioned response (CR) emerges prior to US onset. Converging lines of evidence indicate that the cerebellum, specifically the interpositus nucleus and cerebellar cortex, are essential sites of memory formation and storage for this task (McCormick et al. 1982; McCormick and Thompson 1984a,b; Yeo et al. 1985; Freeman et al. 1995, 2005; Perrett and Mauk 1995; Krupa and Thompson 1997; Garcia and Mauk 1998; Nicholson and Freeman 2002; Jirenhed et al. 2007; Halverson et al. 2010). However, the specific anatomical pathway by which critical auditory CS inputs from cochlear nucleus $(\mathrm{CN})$ reach this cerebellar circuit during learning are currently unclear.

Mossy fiber projections from the pontine nuclei to the cerebellum relay stimulus information from various sensory modalities, including auditory (Steinmetz et al. 1986, 1987; Lewis et al. 1987; Knowlton and Thompson 1988; Tracy et al. 1998; Hesslow et al. 1999; Bao et al. 2000; Halverson and Freeman 2010a,b). The monosynaptic auditory pathway from the $\mathrm{CN}$ to the lateral pontine nuclei (LPN) has been suggested as a possible pathway relaying auditory information to the cerebellum (Steinmetz et al. 1987), but has not been manipulated experimentally. Projections from CN to LPN and other auditory structures are primarily contralateral, the $\mathrm{CN}$ also has polysynaptic paths to additional nuclei within the auditory system which then target LPN (Campolattaro et al. 2007; Halverson et al. 2010). These connections are through auditory structures such as the medial auditory thalamic nuclei (MATN), including the medial division of the medial geniculate

Corresponding author: hunter.halverson@utexas.edu Article is online at http://www.learnmem.org/cgi/doi/10.1101/Im.036947.114.
(MGm), suprageniculate (SG), and posterior interlaminar nucleus (PIN) (Malmierca et al. 2002; Halverson et al. 2010). The unilateral projection from MATN to the LPN is a necessary and sufficient cerebellar input for acquisition and expression of auditory eyeblink conditioning (Halverson and Freeman 2006, 2010a; Campolattaro et al. 2007; Halverson et al. 2008).

In addition to cerebellar learning, the MATN also plays an important role in relaying auditory information to the amygdala during fear conditioning (LeDoux et al. 1984, 1990; McCabe et al. 1993; Campeau and Davis 1995; Poremba and Gabriel 1997). Tetanic stimulation of MATN is sufficient for enhancement of the amygdala-dependent acoustic startle reflex (Huang et al. 2005). Stimulation of the $\mathrm{CN}$ and MATN are also each sufficient for rapid acquisition and expression of eyeblink CRs (Nowak et al. 1999; Campolattaro et al. 2007; Freeman and Duffel 2008; Halverson and Freeman 2010a).

The current experiment used $\mathrm{CN}$ stimulation ipsilateral to the trained eye (left) to increase activity in the contralateral (right) pontine nuclei, relative to a tone stimulus from a previous study (Halverson et al. 2008), in an attempt to provide sufficient input to the cerebellum for eyeblink conditioning during contralateral (right) MATN inactivation. In addition, 2-deoxy-glucose (2-DG) autoradiography was used to examine the metabolic consequences of $\mathrm{CN}$ stimulation combined with MATN inactivation. Increasing the input from $\mathrm{CN}$ to the pontine nuclei, in the absence of MATN input, should support at least partial acquisition or expression of eyeblink conditioning if this source of auditory mossy fiber information is involved with cerebellar learning.

\footnotetext{
(C) 2015 Halverson et al. This article is distributed exclusively by Cold Spring Harbor Laboratory Press for the first 12 months after the full-issue publication date (see http://learnmem.cshlp.org/site/misc/terms.xhtml). After 12 months, it is available under a Creative Commons License (AttributionNonCommercial 4.0 International), as described at http://creativecommons. org/licenses/by-nc/4.0/.
} 


\section{Results}

\section{Cannula and electrode placements}

Cannula placements in the MATN were verified by examining serial coronal sections. All placements $(n=18)$ were in or within $0.5 \mathrm{~mm}$ of the right MGm. Muscimol infusions through cannula placements within this range of the MGm block acquisition and retention of learned eyeblink responses to a tone (Halverson et al. 2008). Figure 1A shows a representative cannula placement in the MATN. All cannula placements were similar to the example shown in Figure 1 with respect to location within the MGm and rostral/caudal extent of the entire MATN.

Electrode placements in the $\mathrm{CN}$ were also verified by examining serial coronal sections. Placements were in the left ventral $(n=$ $13)$ and dorsal $(n=5)$ divisions of the $\mathrm{CN}$. No differences were observed in the rate of learning between rats with dorsal or ventral electrode placements. Figure $1 \mathrm{~B}$ shows a representative electrode placement in the $\mathrm{CN}$.

\section{Eyeblink conditioning}

Acquisition of eyeblink conditioning was tested with left CN stimulation combined with muscimol inactivation of the right MATN (phase 1). Conditioned eyeblink response expression with MATN inactivation was also tested with both stimulation and a tone to identify any differences in response performance between stimulation and a peripheral tone while the MATN was inactivated. If the short-latency (CN to LPN) pathway is involved in sending auditory information to the pontine nuclei sufficient for eyeblink conditioning, maximizing input from the $\mathrm{CN}$ with stimulation during MATN inactivation should support an increase in CR percentage during acquisition, and support at least partial expression of responses during a muscimol retention test by overriding the loss of modulatory input to the LPN from the MATN. However, if direct input from the CN to LPN is not sufficient for cerebellar

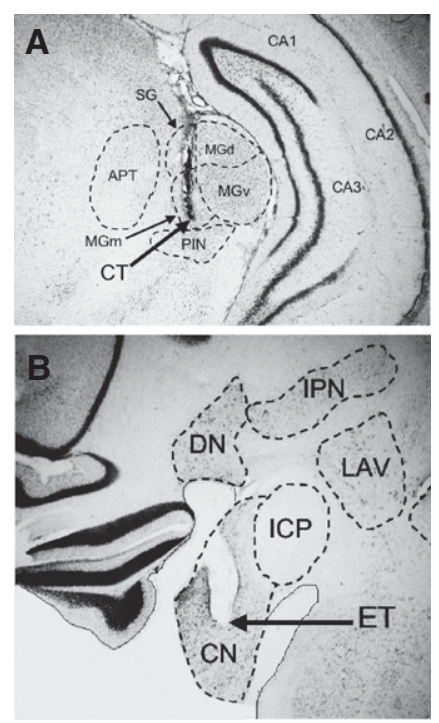

Figure 1. (A) Coronal section of the MATN showing a representative cannula placement in the MGm. (APT) anterior pretectal nucleus, (SG) suprageniculate, (MGm) medial division of the medial geniculate, (MGd) dorsal division of the medial geniculate, (MGv) ventral division of the medial geniculate, (CT) cannula tip. (B) Coronal section of the cerebellum and brainstem showing a representative electrode placement in the CN. (DN) dentate nucleus, (IPN) interpositus nucleus, (LAV) lateral vestibular nucleus, (ICP) inferior cerebellar peduncle, (CN) cochlear nucleus, (ET) electrode tip. Magnification, $2.5 \times$. learning, the muscimol group should fail to learn during infusion sessions, and show a significant decrease in CR percentage during the muscimol retention test despite the facilitation of the $\mathrm{CN}$ to LPN pathway provided by stimulation.

To examine the effects of MATN inactivation during phase 1 on savings in phase 2, a group of control rats were given three sessions of US alone trials (phase 1) followed by three sessions of paired training with cochlear stimulation to compare their subsequent acquisition rate with the muscimol group during postMATN infusion acquisition training (phase 2). The US exposure group controlled for potential sources of behavioral inhibition that could impair CR acquisition rate in the muscimol group during phase 2 as a consequence of blocking auditory information sufficient for learning from reaching the cerebellum with MATN inactivation. If auditory information important for learning is projected to the cerebellum with CN stimulation during MATN inactivation, there should be some evidence of associative learning either during the initial training sessions or during postinfusion training. Alternatively, if auditory information sufficient for learning does not reach the cerebellum in this case, the muscimol and US exposure groups will show identical learning rates during phases 1 and 2 .

Muscimol infusions into the MATN during phase 1 blocked acquisition of conditioned eyeblink responses to $\mathrm{CN}$ stimulation (sessions 1-3; Fig. 2A). The muscimol group showed an identical acquisition rate in phase 2 of training (sessions $4-6$ ) relative to the US exposure control group that received US-alone presentations during phase 1 (Fig. 2A,B). The CR percentage data for the muscimol, saline, and US exposure groups for the three acquisition phases (sessions 1-6 and sessions 9-11) (Fig. 2A,B) were examined with a repeated-measures ANOVA, which revealed an interaction of the group, session, and phase factors, $F_{(8,60)}=17.50, P<$ 0.001 . Tukey's honestly significant difference (HSD) post hoc tests indicated that the saline group produced more CRs than the muscimol and US exposure groups on sessions 1-4 (all comparisons, $P<0.05$ ). The percentage of learned responses did not differ between the muscimol and US exposure groups during the first two phases of training $(P<0.05)$, indicating no associative learning in the muscimol group during phase 1 . The muscimol and US exposure groups also showed significantly more CRs in the second and third sessions of phase 2 (sessions 5-6) and all sessions of phase 3 (sessions 9-11) compared with all sessions of phase 1 (sessions $1-3)(P<0.05$ for each case). This result indicated that conditioning rates during inactivation of MATN while using CN stimulation as a CS were not significantly different from conditioning while receiving presentations of the US alone.

Muscimol infusions into the MATN blocked CRs to both CN stimulation and tone stimuli during the muscimol retention tests (sessions 7 and 12) (Fig. 2A). Repeated-measures ANOVA for both the muscimol retention tests (sessions 6-8, 11-13) revealed a main effect of the session factor for both stimulation, $F_{(2,22)}=$ 552.07, $P<0.0001$, and tone retention tests, $F_{(2,22)}=892.26$, $P<0.0001$ (Fig. 2A). Post hoc tests (HSD) indicated that all rats emitted fewer CRs on the $\mathrm{CN}$ stimulation-muscimol retention test (session 7) than on sessions 6 and $8(P<0.05$ for each case). All rats also emitted fewer CRs on the tone muscimol retention test (session 12) than on sessions 11 and $13(P<0.05)$. These results indicated that inactivation of the MATN with muscimol produced similar impairments in CR frequency with either $\mathrm{CN}$ stimulation or a peripheral tone.

More intense or salient stimuli produce an increase in the rate of acquisition in eyeblink conditioning (Scavio and Gormezano 1974). To examine the saliency of CN stimulation relative to a peripheral tone, the acquisition rates during phases 1 and 2 were compared with acquisition rates of a previous study (Halverson et al. 2008) in which rats received the same training sequence 

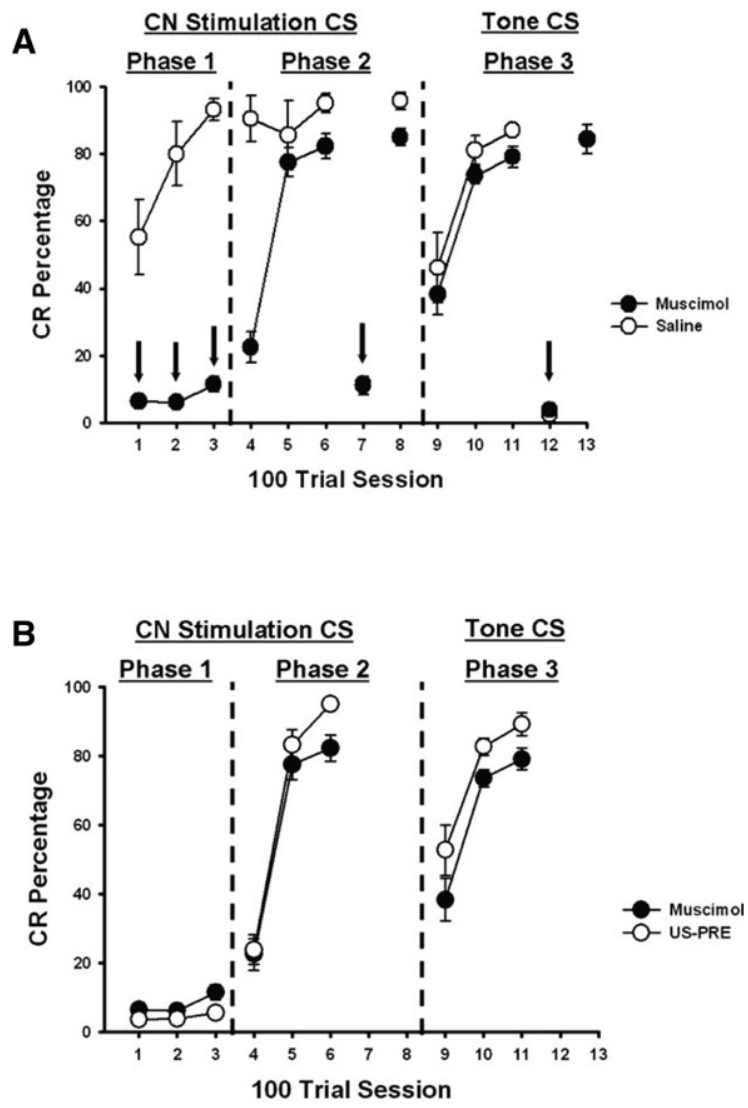

Figure 2. (A) Mean $\pm \mathrm{SE}$ conditioned response (CR) percentage for rats given muscimol (black arrows) or saline during acquisition training (sessions $1-3$ ). All rats received $\mathrm{CN}$ stimulation on sessions $1-8$, and a tone stimulus on sessions 9-13. All rats received muscimol (black arrows) on sessions 7 and 12. Rats given muscimol during acquisition showed no evidence of learning and all rats showed a severe reduction in CRs on the muscimol retention tests (sessions 7 and 12). (B) Mean \pm SE CR percentage for rats given muscimol or US exposure during acquisition training (sessions 1-3). Rats in both groups showed identical acquisition rates on subsequent paired training (sessions 4-6) with $\mathrm{CN}$ stimulation and similar amounts of savings when switched to the tone (sessions 9-11).

(saline or muscimol, followed by no infusions) with the same tone CS used in phase 3 of the current experiment. Repeated-measures ANOVA on the CR percentage data for phases 1 and 2 with CN or tone stimuli revealed a three-way interaction of the phase, session and group factors, $F_{(6,50)}=45.58, P<0.0001$. Tukey's HSD post hoc tests on the data for the saline groups from both experiments indicated that the animals receiving $\mathrm{CN}$ stimulation (current experiment) emitted more CRs on sessions 1 (55.2\% versus $16.9 \%)$ and $2(79.9 \%$ versus $55.9 \%)$ than those receiving the tone (previous experiment) $(P<0.05$ in each case). Post hoc tests on the data for muscimol groups indicated that the animals receiving $\mathrm{CN}$ stimulation showed more CRs on session 5 (77.4\% versus $36.2 \%$ ) than those receiving the tone from the previous study $(P<0.05)$. These results indicated that the $\mathrm{CN}$ stimulation $\mathrm{CS}$ was more salient and supported faster acquisition than the tone CS for both saline and muscimol groups.

Measures of response performance were taken from CS-alone trials to avoid contamination from the cerebellum-independent reflexive response to the US. Response measures were reported on sessions without infusions since the number of CRs during infusion sessions was insufficient for performing statistical analyses. Rats in the muscimol group showed later onset and peak latencies relative to the US exposure and saline groups during the first stimulation session without infusions. Results were examined with repeated-measures ANOVA on the onset and peak latency data for phase 2 which revealed an interaction of the group and session factors for onset, $F_{(4,30)}=6.86, P<0.0001$, and peak latency, $F_{(4,30)}=3.872 ; P<0.012$. Tukey's HSD post hoc tests indicated that rats in the muscimol group showed later onset and peak latencies than both US exposure and saline groups on session 4 $(P<0.05)$. Rats in the US exposure group also showed later CR onsets on session 4 relative to the saline group $(P<0.05)$. This was an expected result since later onset and peak latency observed in the muscimol and US exposure groups is indicative of earlier stages of learning (Gormezano 1972). All three groups showed onset and peak latencies during the first session of tone conditioning (phase 3 , session 9) that indicated new learning to the tone. Results were examined with a repeated-measures ANOVA on the onset and peak latency data, which revealed a main effect of the session factor onset, $F_{(2,30)}=7.00, P<0.003$, and peak latency, $F_{(2,30)}=5.18, P<$ 0.012 . Tukey's HSD post hoc tests revealed that rats in all three groups showed later onset and peak latencies on session 9 than all other tone sessions without infusions $(P<0.05)$.

As animals acquire eyeblink conditioning the amplitude of the response increases in parallel with an increase in CR percentage, which makes changes in amplitude an additional index to investigate learning (Gormezano 1972). Repeated-measures ANOVA on the amplitude data revealed an interaction of the group and session factors for phase $2, F_{(4,30)}=4.27, P<0.007$, and a main effect of the session factor for phase $3, F_{(2,30)}=13.18, P<0.0001$. Post hoc tests (HSD) indicated that the muscimol and US exposure groups showed lower amplitude responses on session 4 relative to the saline group, and the muscimol and US exposure groups showed an increase in response amplitude from session 4 to session $5(P<0.05)$. Post hoc tests also indicated that all three groups showed lower response amplitudes on session 9 than all other tone sessions without infusions during which amplitude was not different between groups ( $P<0.05$ in all cases). The delayed onset and peak latency data combined with the lower response amplitude data serves as evidence that new learning was occurring during the first session when rats were transferred from a CN stimulation CS to the tone.

The startle or $\alpha$ response to CS onset has been classified in rats and mice and is associated with more intense auditory stimulation (Skelton 1988; Boele et al. 2010). This response is triggered by stimulus onset and, unlike the CR, the $\alpha$ response is cerebellar independent (Skelton 1988; Boele et al. 2010). One rat in the saline group consistently showed $\alpha$ responses to very low-intensity $\mathrm{CN}$ stimulation $(5 \mu \mathrm{A})$ even during the muscimol retention test (Fig. 3). Muscimol inactivation of the MATN blocked CRs in this rat but not $\alpha$ responses. All other rats typically showed infrequent small amplitude $\alpha$ responses to $\mathrm{CN}$ stimulation that were attenuated by muscimol infusions into MATN (Fig. 3A,C). Besides the effect on CRs muscimol infusions into MATN or CN stimulation did not produce any overt behavioral changes or ataxia. This result indicates that while low-intensity $\mathrm{CN}$ stimulation can be a highly salient stimulus capable of evoking robust behavioral $\alpha$ responses and rapid CR acquisition, this stimulation is insufficient for the learned cerebellar-dependent response without input from the MATN.

\section{2-DG autoradiography}

Examining CR acquisition rate along with the metabolic effects of both $\mathrm{CN}$ stimulation and tone stimuli in the pontine nuclei under MATN inactivation provided evidence of the increase in stimulus saliency produced by CN stimulation. During presentations of left $\mathrm{CN}$ stimulation metabolic activity in the right MATN was severely 

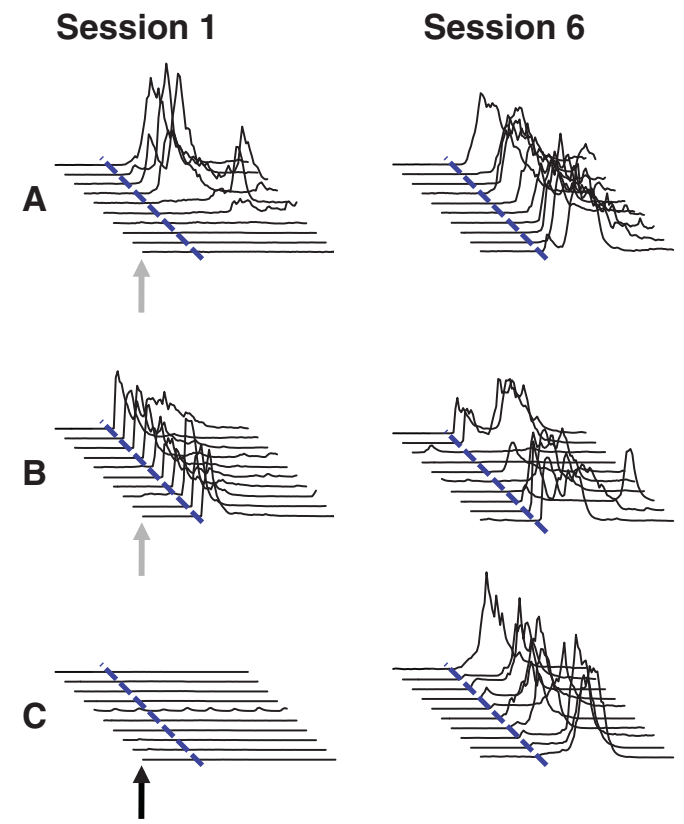

Figure 3. Integrated eyelid EMG traces from CN stimulation-alone trials demonstrating muscimol effects on acquisition (session 1) and expression (sessions 6,7) of conditioned responses. Gray arrows represent saline infusion before training, black arrows represent muscimol infusion before training. Eyelid traces are stacked in order of the session with the first trial in front and the last trial in back of each set. Each trace represents $1 \mathrm{sec}$. $(A)$ Data from a rat in the saline group showing rapid CR acquisition during session 1, CR expression during session 6 followed by CR abolition during MATN muscimol inactivation in session 7. (B) Data from a second rat in the saline group showing a similar pattern of responses as seen in $A$. CN stimulation $(5 \mu \mathrm{A})$ evoked $\alpha$ responses after stimulus onset (dotted blue line) that were not affected by MATN muscimol inactivation. (C) Data from a rat in the muscimol group showing blocked CR acquisition (session 1) and expression (session 7) during MATN inactivation.

reduced in the muscimol group compared with the saline group (Fig. 4). Qualitative assessment of MATN inactivation was done due to the increased 2-DG uptake by gliosis from the cannula track that would confound quantitative readings (Halverson et al. 2008). Metabolic activity in the right auditory cortex was also decreased in the muscimol group relative to the saline group (Fig. 4) most likely due to inactivation of neighboring ventral division of the medial geniculate nucleus (MGv) (Halverson et al. 2008). Bilateral metabolic activity was observed in the $\mathrm{CN}$ in both muscimol and saline groups. The bilateral activity in the $\mathrm{CN}$ was likely reflective of other auditory stimuli that were present during 2-DG uptake including sound from the chamber fan, the rat moving inside the conditioning chamber, and ambient sounds from the laboratory. Bilateral 2-DG uptake was also observed in the superior olive, inferior colliculus, and lateral lemniscus (Fig. 4). Cerebellar activity in the muscimol and saline groups, was similar to the observations with MATN inactivation and presentations of a tone stimulus from a previous study (Halverson et al. 2008) which found no differences between groups. These results replicated the findings of the Halverson et al. (2008) study which used a tone stimulus and inactivation of the right MATN, the focus of this study was on possible differences at the level of the pontine nuclei due to stimulation of the left CN. Region-of-interest measurements were taken from the pontine nuclei to investigate secondary inactivation of the right pontine nuclei relative to the left between the muscimol and saline groups. The right pontine nuclei are important as they are the primary target of both left $\mathrm{CN}$ and right MATN and also the source of auditory mossy fibers necessary for the left cerebellum to learn and express eyeblink CRs. Repeated-measures ANOVA on the normalized region-of-interest 2-DG measurements revealed an interaction of the group and side factors for the pontine nuclei, $F_{(1,7)}=76.98, P<0.001$ (Figs. 4 , 5). Tukey's HSD post hoc tests indicated that the saline group showed more 2-DG uptake in the right and left pontine nuclei than the right side of pons in the muscimol group $(P<0.05)$. Uptake for the left side of the pons in the muscimol group was not significantly different from either side of the saline group $(P>$ 0.05; Figs. 4, 5).

The goal of the current experiment was to increase activity in the pontine nuclei by using $\mathrm{CN}$ stimulation in an attempt to overcome the decrease in pons activity under MATN inactivation observed in a previous study using a tone (Halverson et al. 2008). The 2-DG methods and infusion procedures were the same between the Halverson et al. (2008) study and the current study which allows for direct comparisons to be made between the metabolic activity for rats receiving either $\mathrm{CN}$ stimulation or a tone as the training stimulus. Repeated measures ANOVA on the normalized region-ofinterest 2-DG readings for the pontine nuclei revealed an interaction of the group and side factors, $F_{(3,12)}=26.20$, $P<0.0001$. HSD post hoc tests indicated that rats receiving $\mathrm{CN}$ stimulation had more 2-DG uptake on both sides of the pontine nuclei than rats receiving the tone in both groups, saline (left $=167.8$ $\mathrm{nCi} / \mathrm{g}$ versus 134.1 , right $=174.3 \mathrm{nCi} / \mathrm{g}$ versus 134.7) and muscimol (left $=166.9 \mathrm{nCi} / \mathrm{g}$ versus 118.5 . right $=115.5 \mathrm{nCi} / \mathrm{g}$ versus 67.4 $)(P<0.05)$. This result indicates that $\mathrm{CN}$ stimulation effectively increased the metabolic activity in the pontine nuclei, even during MATN inactivation, above the level observed with a tone CS.

\section{Discussion}

Acquisition and expression of cerebellar-dependent eyeblink CRs with CN stimulation ipsilateral to the trained eye (left) were severely impaired by muscimol inactivation of the contralateral (right) MATN. Retention of CRs with a tone CS was also severely impaired by muscimol inactivation of the MATN, as seen in a previous study (Halverson et al. 2008). Rats in the muscimol group showed no evidence of associative learning during initial acquisition in phase 1 (Fig. 2A, sessions 1-3) and a significantly impaired acquisition rate during post-infusion training in phase 2 (Fig. 2A, sessions 4-6) that was very similar to the US exposure control group (Fig. 2B). A muscimol retention test (Fig. 2A, sessions 7 and 12) blocked CRs in all rats with both CN stimulation and tone stimuli, which confirmed similar cannula placements in the MATN between the saline and muscimol groups. Metabolic activity in the MATN was severely reduced in the muscimol group compared with animals that received saline infusions (Fig. 4). Metabolic activity in the right pontine nuclei was also significantly reduced relative to the left side of pons in the muscimol group and in relation to the metabolic activity observed in the saline group (Figs. 4, 5).

The rapid CR acquisition rate observed with $\mathrm{CN}$ stimulation relative to a previous study using a tone CS (Halverson et al. 2008) 


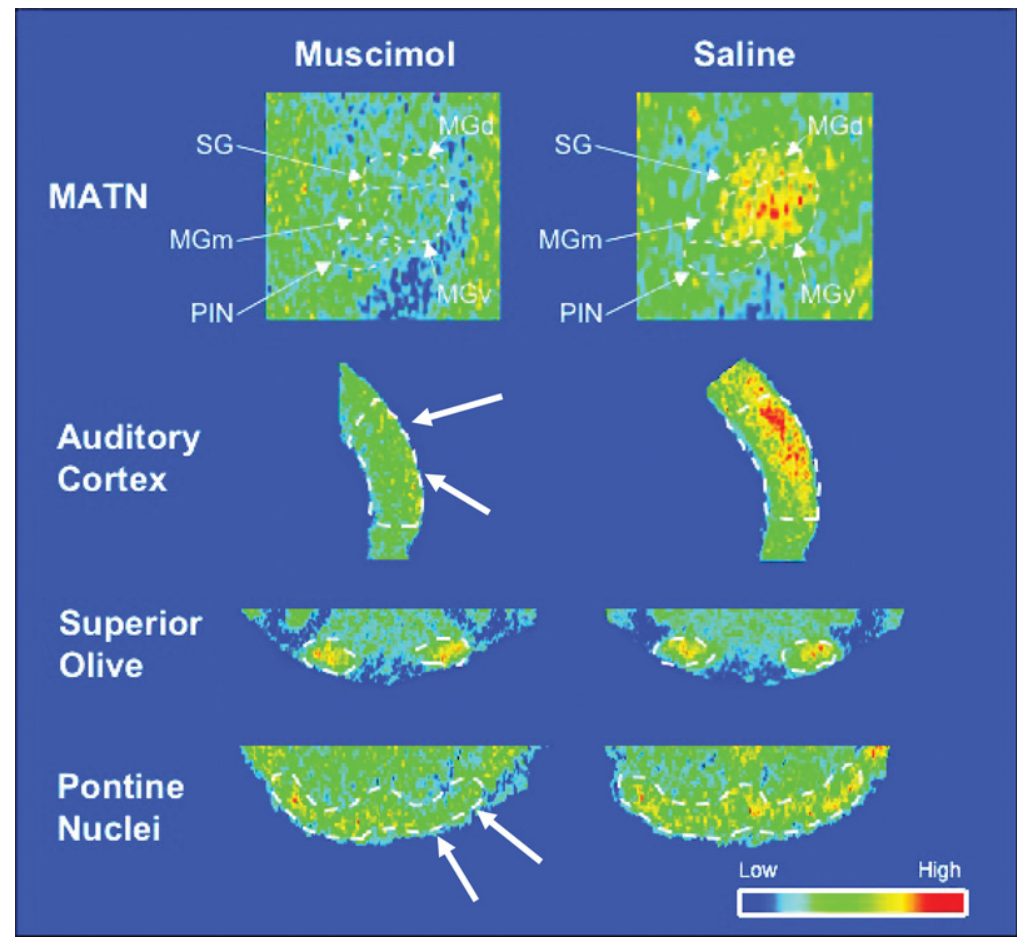

Figure 4. Autoradiographs showing uptake of 2-DG radiolabeled with ${ }^{14} \mathrm{C}$ (red indicates strongest 2-DG uptake). Dotted lines: Boundaries of the medial auditory thalamic nuclei (MATN), auditory cortex, superior olive, and pontine nuclei. Boundaries were drawn based on the same thionin-stained sections. Upper row: Representative MATN sections showing activity in the auditory thalamus in the muscimol and saline groups. Second row: auditory cortex; third row: superior olive; lower row: pontine sections show secondary inactivation (white arrows) after ipsilateral muscimol infusion and saline infusions into MATN.

suggests that $\mathrm{CN}$ stimulation serves as a very salient stimulus. Stimulation of the CN could possibly increase activity in the pontine nuclei (relative to a tone CS) to a level sufficient to overcome MATN inactivation and support learning. The use of left CN stimulation produced a differential unilateral increase in metabolic activity in the right pontine nuclei during inactivation of the right MATN compared with the metabolic activity observed in the same side of pons while using the tone stimulus from the Halverson et al. (2008) study. This increase provided by CN stimulation in the direct input from $\mathrm{CN}$ to the contralateral pontine nuclei would have likely provided adequate input to the cerebellum for at least partial acquisition or expression of eyeblink conditioning during MATN inactivation. Inactivation of the right MATN produced a decrease in metabolic activity in the pons in this study, this decrease in activity was likely due to decreased input from both MATN and auditory cortex (Fig. 4), since both areas showed decreases in 2-DG uptake during MATN inactivation (Halverson et al. 2008).

Rats were unable to acquire or express conditioned eyeblink responses with ipsilateral $\mathrm{CN}$ stimulation under contralateral MATN inactivation. This result provides compelling evidence that information in the auditory system must be relayed through the MATN to the pontine nuclei for both acquisition and expression of cerebellar-dependent eyeblink CRs. It is still possible that the direct CN to LPN projection is somehow involved with sending auditory information to the cerebellum for learning. This projection will need to be silenced during eyeblink conditioning using a technique like optogenetics to rule out any involvement with cerebellar learning. Future studies may also extend this auditory-evoked engagement of MATN to associative learning in general. Muscimol inactivation during the initial acquisition sessions (sessions 1-3) also slowed acquisition rates in the subsequent post-muscimol training (session 4) relative to the first session in phase 1 of the saline group. A control group that initially received three USalone sessions displayed a similar delay in CR acquisition rate on the first paired session with cochlear stimulation (Fig. 3 , session 4). This result provides evidence that blocking auditory stimulation at the level of the MATN during paired training is similar to training with presentations of the US alone at the level of the cerebellum. The increased input from the $\mathrm{CN}$ to the pontine nuclei and cerebellum was not sufficient to support acquisition or expression of CRs in the absence of input from the MATN. Unlike the learned cerebellar-dependent CRs, startle responses elicited by $\mathrm{CN}$ stimulation in one rat were not blocked by MATN inactivation (Fig. 3B), smaller startle responses in all other rats were typically attenuated during MATN inactivation. Thus, MATN inactivation selectively blocked the auditory information sufficient for CR acquisition and expression from being relayed to the pontine nuclei and into the cerebellum.

The results of this study provide additional evidence that convergent auditory inputs to the MATN contralateral to the trained eye and the corresponding ipsilateral MATN projection to the LPN comprise the necessary auditory pathway for acquisition and expression of cerebellar-dependent eyeblink CRs to auditory stimuli (Fig. 6; Halverson and Freeman 2006, 2010a; Campolattaro et al. 2007; Freeman et al. 2007; Halverson et al. 2008). The laterality of $\mathrm{CN}$ stimulation and MATN inactivation in the current experiment was selected based on the connectivity within the auditory system and how the auditory system interfaces with the eyeblink

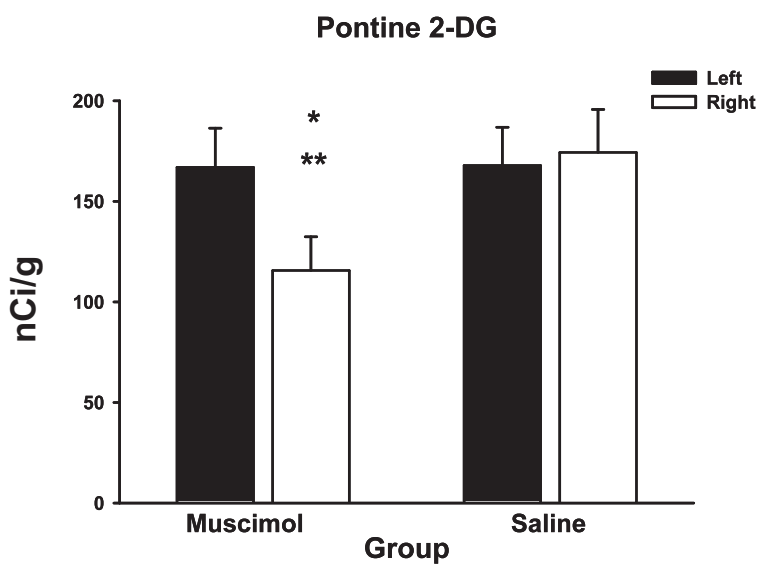

Figure 5. Mean $\pm \mathrm{SE} \mathrm{nCi} / \mathrm{g}$ of 2-DG uptake in the left and right side of the basal pontine nuclei in the muscimol and saline groups. $\left(^{*}\right)$ Significant difference $(P<0.05)$ between the muscimol and saline groups; $\left({ }^{* *}\right)$ significant difference $(P<0.05)$ between left and right side within groups. 


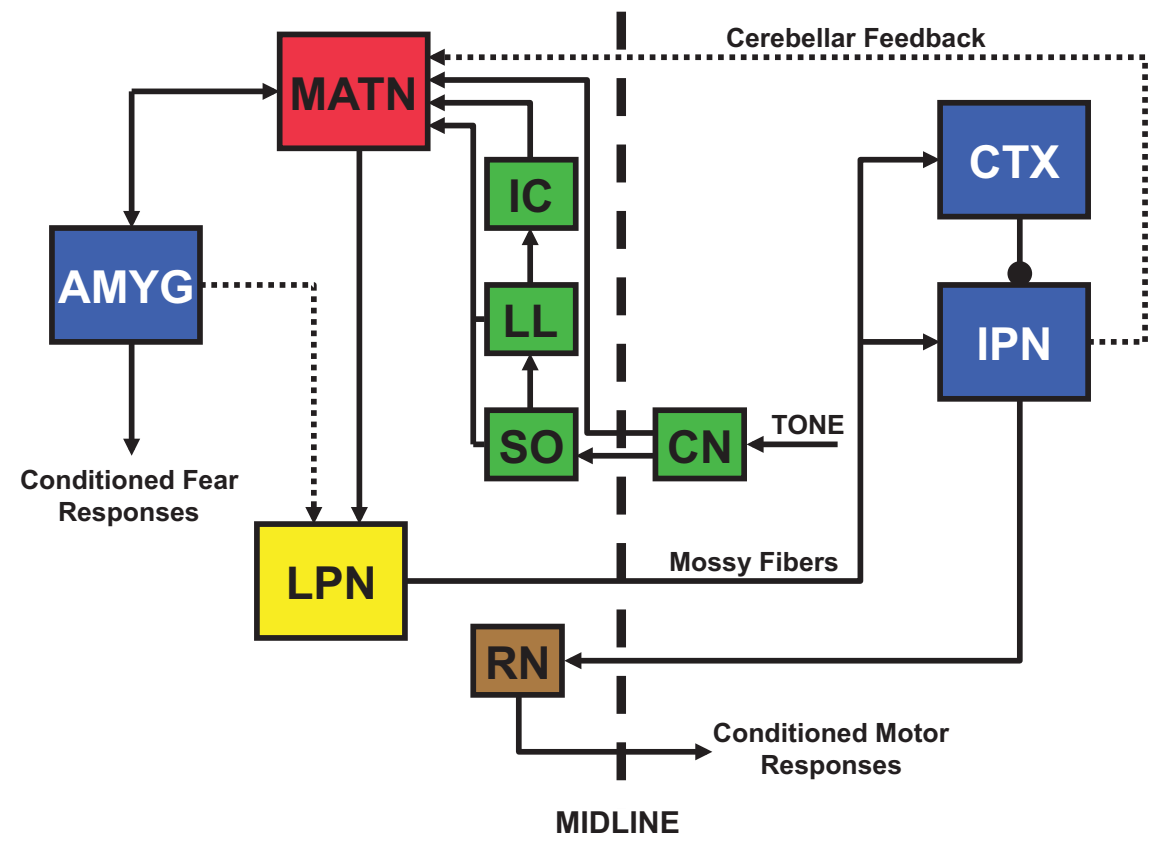

Figure 6. The auditory pathway for associative fear and cerebellar-dependent motor learning. Parallel inputs into the MATN from contralateral cochlear nucleus (CN), and primarily unilateral inputs from superior olive (SO), lateral lemniscus (LL), and inferior colliculus (IC). Direct unilateral projection from MATN to the LPN. LPN mossy fiber projection into the contralateral cerebellum (cortex [CTX] and interpositus nucleus [IPN]), and IPN output to red nucleus (RN) for CR expression, IPN also has feedback to MATN. The MATN also has a reciprocal connection with the amygdala (AMYG), which is necessary for fear and avoidance learning. The AMYG also projects to LPN and could modulate cerebellar-dependent motor learning via this pathway. Blue boxes indicate sites of memory formation and storage. Dotted lines indicate modulatory inputs.

conditioning circuitry (LPN). Stimulation of CN bilaterally or even contralateral to the trained eye was not used in the current experiment due to the connections in the auditory system and how auditory structures project onto the known eyeblink conditioning circuitry highlighted above and illustrated in Figure 6. Contralateral (right) $\mathrm{CN}$ stimulation would likely be a sufficient $\mathrm{CS}$ for eyeblink conditioning, however, because $\mathrm{CN}$ has primarily contralateral projections to higher auditory structures and to LPN, stimulating the right $\mathrm{CN}$ would not engage the eyeblink conditioning circuitry as directly as the stimulation used in the current experiment.

Learning-dependent cerebellar feedback to MATN may enhance thalamic output to the LPN related to the stimulus used in training and facilitate further plasticity in the cerebellum (Halverson et al. 2010). The MATN, along with auditory cortex, is also part of the auditory pathway necessary for amygdaladependent fear conditioning (Romanski and LeDoux 1992). Stimulation of the auditory cortex is a sufficient CS for eyeblink conditioning (Knowlton and Thompson 1992; Nowak et al. 1999). However, lesions of the entire cerebral cortex (including auditory cortex) do not prevent acquisition or retention of eyeblink conditioning to an auditory stimulus (Oakley and Russell 1972, 1977). In addition to the MATN being important for the relay of auditory information to the cerebellum and amygdala, both fear and eyeblink conditioning produce short-latency plasticity (>50 msec) in MATN within the first few learning trials (Gabriel et al. 1975; Quirk et al. 1995; Maren et al. 2001; Poremba and Gabriel 2001; Halverson et al. 2010). The many similarities between cerebellar-dependent motor and amygdala-dependent fear conditioning have been reviewed in detail while identifying different auditory pathways for both types of learning (Medina et al. 2002; Fanselow and Poulos 2005; Boele et al. 2010). Appetitive conditioning with an auditory stimulus also leads to learning-related potentiation in auditory thalamic firing (Disterhoft and Olds 1972; Nienhuis and Olds 1978), but has not been investigated to the same extent as thalamic involvement in eyeblink or fear conditioning. Acquisition of eyeblink or fear conditioning can enhance conditioning in the other paradigm while using the same auditory stimulus. This may be due to learning-related changes in the MATN that are established during the initial phase of learning that then subsequently facilitates MATN input important for later learning in the other paradigm (Neufeld and Mintz 2001; Lindquist et al. 2010). Similar to eyeblink conditioning, if the US used in fear conditioning is unilateral (e.g., eye shock) the MATN and amygdala engaged in learning and expressing conditioned fear responses are also unilateral (Fig. 6; LeDoux et al. 1991; Blair et al. 2005). Amygdala modulation of cerebellar-dependent motor learning could therefore be influenced from either the direct amygdala projection to LPN or from the reciprocal connection between the amygdala and MATN (Prince and Amaral 1981; Maren et al. 2001; Poremba and Gabriel 2001; Taub and Mintz 2010; Ng and Freeman 2013).

Ipsilateral (to the trained eye) $\mathrm{CN}$ stimulation was used in an attempt to increase the activity in the $\mathrm{CN}$ projection to the contralateral pons during eyeblink conditioning and possibly overcome the effect of MATN inactivation on CR acquisition and/or expression. Although increased activity in the pontine nuclei was observed with $\mathrm{CN}$ stimulation (relative to a tone), rats did not acquire or express CRs under MATN inactivation. The MATN is the necessary auditory input to the LPN for cerebellar-dependent eyeblink conditioning and is also engaged in many other associative learning paradigms including appetitive, avoidance, and fear learning. The MATN may, therefore, be a centerpiece of auditory associative learning regardless of the reinforcing events or responses measured.

\section{Materials and Methods}

\section{Subjects}

The subjects were 18 male Long-Evans rats (250-400 g). The rats were housed in the animal colony in Spence Laboratories of Psychology at the University of Iowa (Iowa City, IA). All rats were maintained on a $12 \mathrm{~h}$ light-dark cycle and given ad libitum access to food and water. All procedures involving animals were approved by the University of Iowa Institutional Animal Care and Use Committee.

\section{Surgery}

One week before training, rats were removed from their home cages and anesthetized with isoflurane. After anesthesia onset, rats were fitted with differential electromyograph (EMG) electrodes (stainless steel) implanted into the upper left orbicularis oculi muscle. The reference electrode was a silver wire attached to a stainless steel skull screw. The EMG electrode leads terminated in 
gold pins in a plastic connecter. A bipolar stimulating electrode (Plastics One) for shock US delivery was implanted subdermally, caudal to the left eye. A 23-gauge guide cannula was implanted $2.0 \mathrm{~mm}$ dorsal to the right MGm. A 30-gauge stylet was inserted into the guide cannula and extended $1.0 \mathrm{~mm}$ from the end of the guide. Stereotaxic coordinates for the guide cannula were $5.5 \mathrm{~mm}$ posterior to bregma, $3.1 \mathrm{~mm}$ lateral to midline, and 6.3 $\mathrm{mm}$ ventral to skull surface. A bipolar stimulating electrode was implanted into the left dorsal or ventral $\mathrm{CN}(10.9 \mathrm{~mm}$ posterior to bregma, $3.8 \mathrm{~mm}$ lateral to midline, and $8.4 \mathrm{~mm}$ ventral to skull surface). The plastic connector housing the EMG electrode leads, both bipolar stimulating electrodes, the guide cannula, and three skull screws were secured to the skull with Osteobond copolymer bone cement (Zimmer). Animals were maintained on $0.006 \%$ Sulfatrim (Hi-Tech Pharmacal Co.) in water for $4 \mathrm{~d}$ after surgery.

\section{Muscimol infusion procedure}

The $\gamma$-aminobutyric acid (GABA) receptor agonist muscimol was used to create a temporary localized lesion by hyperpolarizing cells around the infusion site. Cells that are hyperpolarized by muscimol are less likely to fire action potentials and remain in that inactivated state until the drug is no longer effective (Majchrzak and Di Scala 2000). Muscimol binds with the GABA receptor, which allows chloride ions to enter the cell, effectively decreasing neural activity beginning $30 \mathrm{~min}$ after infusion with the effect lasting at least $2 \mathrm{~h}$ post-infusion (Martin 1991).

Before the muscimol infusions, the stylet was removed from the guide cannula and replaced with a 30-gauge infusion cannula that extended $2.0 \mathrm{~mm}$ beyond the guide cannula. The infusion cannula was connected to polyethylene tubing (PE 10; 110-120 $\mathrm{cm}$ ), which was connected to a $10 \mu \mathrm{L}$ gas tight syringe (Hamilton). The syringe was placed in an infusion pump (Harvard Apparatus), and $1.0 \mu \mathrm{L}$ of muscimol $(10 \mathrm{mM}, \mathrm{pH}=7.4)$ or saline was infused over $2 \mathrm{~min}$ at a rate of $30 \mu \mathrm{L} / \mathrm{h}$. The large infusion volume $(1.0$ $\mu \mathrm{L}$ ) was used to target all the nuclei of the MATN (MGm, PIN, and SG) with a single infusion. After the infusion, the tubing connected to the infusion cannula was cut and sealed with candle wax. The infusion cannula remained in place for the duration of the experimental session and was replaced by the stylet after each session.

\section{Apparatus}

The conditioning apparatus consisted of two small sound-attenuating chambers (BRS/LVE). Within each sound-attenuating chamber was a small-animal operant chamber (BRS/LVE) in which the rats were kept during conditioning. One wall of the operant chamber was fitted with two speakers that independently produced tones of up to $120 \mathrm{~dB}$ with a $\sim 1000-9000 \mathrm{~Hz}$ frequency range. A wall-mounted exhaust fan provided a $65 \mathrm{~dB}$ masking noise. The $\mathrm{CN}$ stimulation used in training was a $300 \mathrm{msec}$ train of $0.1-\mathrm{msec}$ biphasic square wave pulses $(5-50 \mu \mathrm{A})$ at $50 \mathrm{~Hz}$. Stimulation level was independently set for each rat by starting at $50 \mu \mathrm{A}$ and determining the intensity that produced a behavioral response before training, then reducing the current in $5 \mu \mathrm{A}$ increments until no behavioral response was observed. This individual stimulation level for each rat was then used throughout the experiment. Typical behavioral responses to $\mathrm{CN}$ stimulation were head turns, ear/whisker movement, and rearing. One rat displayed $\alpha$ or startle responses to $\mathrm{CN}$ stimulation at $5 \mu \mathrm{A}$ and was trained at this low-intensity level to investigate the effect of MATN inactivation on the stimulation elicited $\alpha$ responses. The tone stimulus used in training was a $2000 \mathrm{~Hz}$ pure tone $(85 \mathrm{~dB}$; range in conditioning chamber, $83-87 \mathrm{~dB}$ ). The electrode leads from the rat's headstage were connected to peripheral equipment by lightweight cables that allowed the rat to move freely during conditioning. A desktop computer was connected to the peripheral equipment. Computer software controlled the delivery of stimuli and the recording of eyelid EMG activity (JSA Designs). The periorbital shock stimulus was delivered through a stimulus isolator (model 365A; World Precision Instruments). Eyelid EMG activity was amplified differentially (gain, 2000; sampling rate,
$250 \mathrm{~Hz}$ ), filtered (500-5000 Hz), and integrated (time constant, $20 \mathrm{msec}$ ). The intensity of the shock US was set at two times the threshold for eliciting a discrete eyeblink (typical range of final current intensity, 2-4 mA; $60 \mathrm{~Hz}$; constant current). Current intensity of the US was determined during the first few training trails of the first day of training and held at that level throughout training for each rat.

\section{Conditioning procedure}

Rats adapted to the training environment for $5 \mathrm{~min}$ before each training session. During phase 1 , saline $(n=6)$ or muscimol $(n=7)$ was infused into the MATN 30 min prior to each of three delay eyeblink conditioning sessions with each session consisting of 100 trials per day. Trials were presented with a pseudorandom distribution of intertrial intervals between 18 and $42 \mathrm{sec}$ (30 sec average), in which $300 \mathrm{msec} C \mathrm{~N}$ stimulation coterminated with a 25-msec shock US, yielding an interstimulus interval of 275 msec. Each of the first three sessions was separated by $3 \mathrm{~d}$ to prevent tolerance to muscimol. Following the three infusion sessions all rats were given three daily sessions without infusions in order to examine the amount of savings from the initial training (phase 2). Behavioral savings observed in the muscimol group during the post-infusion sessions would indicate acquisition of associative learning during the initial infusion sessions even though performance was impaired. All rats then received a final infusion of muscimol followed by a recovery session. Following training with $\mathrm{CN}$ stimulation, all rats received three sessions of delay conditioning with the tone ( $300 \mathrm{msec}, 2 \mathrm{kHz}$ ) and shock US (phase 3). Following these three sessions, all rats received an additional infusion of muscimol followed by a recovery session. A control group $(n=$ 5 ) was given three sessions of US alone presentations (phase 1) followed by three sessions of paired training with $\mathrm{CN}$ stimulation (phase 2), and finally three sessions with the tone (phase 3). The US exposure group was used to determine whether muscimol inactivation of the MATN was behaviorally equivalent to presenting no conditioned stimulus during training. This is particularly important for assessing performance during phase 2 because a difference in savings between the saline and muscimol groups could be affected by US exposure, context exposure, or context conditioning in addition to differences in learning during phase 1. Daily training sessions consisted of 10 blocks of nine paired CN stimulation or tone-US presentations followed by a CN stimulation or tone alone trial. The values relayed to the computer software from the EMG integrator were voltage values of integrated EMG activity. The CR threshold was set to $0.4 \mathrm{~V}$ above the amplified and integrated EMG activity at baseline. The EMG baseline was typically zero. Integrated EMG responses that exceeded the threshold value during the first $80 \mathrm{msec}$ of stimulus onset were considered startle or $\alpha$ responses; responses that exceeded the threshold value during the last $195 \mathrm{msec}$ of the CS were considered CRs; responses that crossed the threshold after US onset were defined as unconditioned responses.

\section{Histology}

After training, the rats were killed with a lethal injection of sodium pentobarbital $(150 \mathrm{mg} / \mathrm{kg})$ and transcardially perfused with $100 \mathrm{~mL}$ of physiological saline followed by $300 \mathrm{~mL}$ of $10 \%$ neutral buffered formalin (Surgipath). After perfusion, the brains were post-fixed in the same fixative for a minimum of $24 \mathrm{~h}$, cryoprotected in a $30 \%$ sucrose in formalin solution, and subsequently sectioned at $50 \mu \mathrm{m}$ with a sliding microtome. Sections were stained with thionin. The locations of the cannula and electrode placements were verified using a light microscope (Leica DMLS, Wetzlar, Germany) and stereotaxic brain atlas (Paxinos and Watson 1998).

\section{2-DG procedure and analysis}

After training, some of the rats were given saline $(n=5)$ or muscimol $(n=7)$ infusions into the MATN 30 min before exposure to the $\mathrm{CN}$ stimulation used in training. Twenty minutes after the 
infusion, each rat received an intraperitoneal injection of a glucose analog, 2-DG radiolabeled with ${ }^{14} \mathrm{C}(20 \mu \mathrm{Ci} / 100 \mathrm{~g}$ of body weight; American Radiolabeled Chemicals) in $0.3 \mathrm{~mL}$ of sterile saline. After the injection the rats were put into the training chamber and presented with $60 \mathrm{CN}$ stimulation-alone trials during the 45 min of 2-DG uptake. Rats were immediately decapitated, brains were quickly removed, frozen at $-37^{\circ} \mathrm{C}$, and stored at $-80^{\circ} \mathrm{C}$. Brains were sectioned on a cryostat at $40 \mu \mathrm{m}$. Serial sections were quick dried on glass coverslips, glued on poster board, and exposed to ${ }^{14} \mathrm{C}$ phosphor imaging screens for $36 \mathrm{~h}$ along with ${ }^{14} \mathrm{C}$ standards (Amersham Biosciences). The screens were read and digitized by a phosphor imaging system (Cyclone Storage Phosphor System; PerkinElmer Life Sciences). Individual calibration curves were calculated based on absolute gray levels of the ${ }^{14} \mathrm{C}$ standards on each film. Subsequent densitometric measures were then automatically converted to units of nanoCuries per gram (nCi/g). Region-of-interest measurements (ImageJ, NIH) were taken from the pontine nuclei to examine the secondary effects of MATN inactivation during CN stimulation. Measurements were taken from the calibrated film by tracing around each side of the pontine nuclei throughout its extent. The 2-DG techniques were similar to previous studies that examined metabolic activity of the cerebellum and MATN after inactivation with muscimol (Freeman et al. 2005; Halverson et al. 2008). This mapping technique has previously been used to identify possible metabolic differences in the cerebellum during trace and delay eyeblink conditioning, and also to explore metabolic differences in various neural structures including thalamus and auditory cortex during conditioned bradycardia (Gonzalez-Lima and Scheich 1986a,b; Plakke et al. 2007).

\section{Acknowledgments}

This research was supported by National Institute for Mental Health grant MH080005. We thank Loren C. Hoffmann for her helpful suggestions with preparing the manuscript.

\section{References}

Bao S, Chen L, Thompson RF. 2000. Learning- and cerebellum-dependent neuronal activity in the lateral pontine nucleus. Behav Neurosci 114: $254-261$.

Blair HT, Huynh VK, Vaz VT, Van J, Patel RR, Hiteshi AK, Lee JE, Tarpley JW. 2005. Unilateral storage of fear memories by the amygdala. J Neurosci 25: 4198-4205.

Boele HJ, Koekkoek SK, De Zeeuw CI. 2010. Cerebellar and extracerebellar involvement in mouse eyeblink conditioning: the ACDC model. Front Cell Neurosci 3: 19.

Campeau S, Davis M. 1995. Involvement of subcortical and cortical afferents to the lateral nucleus of the amygdala in fear conditioning measured with fear-potentiated startle in rats trained with auditory and visual conditioned stimuli. J Neurosci 15: 2312-2327.

Campolattaro MM, Halverson HE, Freeman JH. 2007. Medial auditory thalamic stimulation as a conditioned stimulus for eyeblink conditioning in rats. Learn Mem 14: 152-159.

Christian KM, Thompson RF. 2003. Neural substrates of eyeblink conditioning: acquisition and retention. Learn Mem 10: 427-455.

Disterhoft JF, Olds J. 1972. Differential development of conditioned unit changes in thalamus and cortex of rat. J Neurophysiol 35: 665-679.

Fanselow MS, Poulos AM. 2005. The neuroscience of mammalian associative learning. Annu Rev Psychol 56: 207-234.

Freeman JH, Duffel JW. 2008. Eyeblink conditioning using cochlear nucleus stimulation as a conditioned stimulus in developing rats. Dev Psychobiol 50: 640-646.

Freeman JH, Steinmetz AB. 2011. Neural circuitry and plasticity mechanisms underlying delay eyeblink conditioning. Learn Mem 18: 666-677.

Freeman JH Jr, Carter CS, Stanton ME. 1995. Early cerebellar lesions impair eyeblink conditioning in developing rats: differential effects of unilateral lesions on postnatal day 10 or 20. Behav Neurosci 109: 893-902.

Freeman JH Jr, Halverson HE, Poremba A. 2005. Differential effects of cerebellar inactivation on eyeblink conditioned excitation and inhibition. J Neurosci 25: 889-895.

Freeman JH, Halverson HE, Hubbard EM. 2007. Inferior colliculus lesions impair eyeblink conditioning in rats. Learn Mem 14: 842-846.
Gabriel M, Saltwick SE, Miller JD. 1975. Conditioning and reversal of short-latency multiple-unit responses in the rabbit medial geniculate nucleus. Science 189: 1108-1109.

Garcia KS, Mauk MD. 1998. Pharmacological analysis of cerebellar contributions to the timing and expression of conditioned eyelid responses. Neuropharmacology 37: 471-480.

Gonzalez-Lima F, Scheich H. 1986a. Classical conditioning of tone-signaled bradycardia modifies 2-deoxyglucose uptake patterns in cortex, thalamus, habenula, caudate-putamen and hippocampal formation. Brain Res 363: 239-256.

Gonzalez-Lima F, Scheich H. 1986b. Neural substrates for tone-conditioned bradycardia demonstrated with 2-deoxyglucose. II. Auditory cortex plasticity. Behav Brain Res 20: 281-293.

Gormezano I. 1972. Investigations of defense and reward conditioning in the rabbit. Classical Conditioning II: Current Research and Theory. Appleton, New York.

Gormezano I, Kehoe EJ, Marshall BS. 1983. Twenty years of classical conditioning with the rabbit. Progr Psychobiol Physiol Psychol 10: $197-275$.

Halverson HE, Freeman JH. 2006. Medial auditory thalamic nuclei are necessary for eyeblink conditioning. Behav Neurosci 120: 880-887.

Halverson HE, Freeman JH. 2010a. Medial auditory thalamic input to the lateral pontine nuclei is necessary for auditory eyeblink conditioning. Neurobiol Learn Mem 93: 92-98.

Halverson HE, Freeman JH. 2010b. Ventral lateral geniculate input to the medial pons is necessary for visual eyeblink conditioning in rats. Learn Mem 17: 80-85.

Halverson HE, Poremba A, Freeman JH. 2008. Medial auditory thalamus inactivation prevents acquisition and retention of eyeblink conditioning. Learn Mem 15: 532-538.

Halverson HE, Lee I, Freeman JH. 2010. Associative plasticity in the medial auditory thalamus and cerebellar interpositus nucleus during eyeblink conditioning. J Neurosci 30: 8787-8796.

Hesslow G, Svensson P, Ivarsson M. 1999. Learned movements elicited by direct stimulation of cerebellar mossy fiber afferent. Neuron 24: $179-185$

Huang J, Wu X, Yeomans J, Li L. 2005. Opposite effects of tetanic stimulation of the auditory thalamus or auditory cortex on the acoustic startle reflex in awake rats. Eur J Neruosci 21: 1943-1956.

Jirenhed DA, Bengtsson F, Hesslow G. 2007. Acquisition, extinction, and reacquisition of a cerebellar cortical memory trace. J Neurosci 27: 2493-2502.

Knowlton BJ, Thompson RF. 1988. Microinjections of local anesthetic into the pontine nuclei reduce the amplitude of the classically conditioned eyelid response. Physiol Behav 43: 855-857.

Knowlton BJ, Thompson RF. 1992. Conditioning using a cerebral cortical conditioned stimulus is dependent on the cerebellum and brain stem circuitry. Behav Neurosci 106: 509-517.

Krupa DJ, Thompson RF. 1997. Reversible inactivation of the cerebellar interpositus nucleus completely prevents acquisition of the classically conditioned eyeblink response. Learn Mem 3: 545-556.

LeDoux JE, Sakaguchi A, Reis DJ. 1984. Subcortical efferent projections of the medial geniculate nucleus mediate emotional responses conditioned to acoustic stimuli. J Neurosci 4: 683-698.

LeDoux JE, Farb C, Ruggiero DA. 1990. Topographic organization of neurons in the acoustic thalamus that project to the amygdala. $J$ Neurosci 10: 1043-1054.

LeDoux JE, Farb CR, Milner TA. 1991. Ultrastructure and synaptic associations of auditory thalamo-amygdala projections in the rat. Exp Brain Res 85: 577-586.

Lewis LJ, Lo Turco JJ, Solomon PR. 1987. Lesions of the middle cerebellar peduncle disrupt acquisition and retention of the rabbit's classically conditioned nictitating membrane response. Behav Neurosci 101: $151-157$

Lindquist DH, Mahoney LP, Steinmetz JE. 2010. Conditioned fear in adult rats is facilitated by the prior acquisition of a classically conditioned motor response. Neurobiol Learn Mem 94: 167-175.

Majchrzak M, Di Scala G. 2000. GABA and muscimol as reversible inactivation tools in learning and memory. Neural Plast 7: 19-29.

Malmierca MS, Merchán MA, Henkel CK, Oliver DL. 2002. Direct projections from cochlear nuclear complex to auditory thalamus in the rat. J Neurosci 22: 10891-10897.

Maren S, Yap SA, Goosens KA. 2001. The amygdala is essential for the development of neuronal plasticity in the medial geniculate nucleus during auditory fear conditioning in rats. J Neurosci 21: RC135.

Martin JH. 1991. Autoradiographic estimation of the extent of reversible inactivation produced by microinjection of lidocaine and muscimol in the rat. Neurosci Lett 127: 160-164.

McCabe PM, McEchron MD, Green EJ, Schneiderman N. 1993. Electrolytic and ibotenic acid lesions of the medial subnucleus of the medial geniculate prevent the acquisition of classically conditioned heart rate to a single acoustic stimulus in rabbits. Brain Res 619: 291-298. 
McCormick DA, Thompson RF. 1984a. Neuronal responses of the rabbit cerebellum during acquisition and performance of a classically conditioned nictitating membrane-eyelid response. J Neurosci 4: $2811-2822$.

McCormick DA, Thompson RF. 1984b. Cerebellum: essential involvement in the classically conditioned eyelid response. Science 223: 296-299.

McCormick DA, Clark GA, Lavond DG, Thompson RF. 1982. Initial localization of the memory trace for a basic form of learning. Proc Natl Acad Sci 79: 2731-2735.

Medina JF, Repa JC, Mauk MD, LeDoux JE. 2002. Parallels between cerebellum- and amygdala-dependent conditioning. Nat Rev Neurosci 3: $122-131$.

Nicholson DA, Freeman JH Jr. 2002. Neuronal correlates of conditioned inhibition of the eyeblink response in the anterior interpositus nucleus. Behav Neurosci 116: 22-36.

Neufeld M, Mintz M. 2001. Involvement of the amygdala in classical conditioning of eyeblink response in the rat. Brain Res 889: 112-117.

Nienhuis R, Olds J. 1978. Changes in unit responses to tones after food reinforcement in the auditory pathway of the rat: intertrial arousal. Exp Neurol 59: 229-242.

$\mathrm{Ng} \mathrm{KH}$, Freeman JH. 2013. Amygdala inactivation impairs eyeblink conditioning in developing rats. Dev Psychobiol 56: 999-1007.

Nowak AJ, Kehoe EJ, Macrae M, Gormezano I. 1999. Conditioning and reflex modification of the rabbit nictitating membrane response using electrical stimulation in auditory nuclei. Behav Brain Res 105: 189-198.

Oakley DA, Russell IS. 1972. Neocortical lesions and Pavlovian conditioning. Physiol Behav 8: 915-926.

Oakley DA, Russell IS. 1977. Subcortical storage of Pavlovian conditioning in the rabbit. Physiol Behav 18: 931-937.

Paxinos G, Watson C. 1998. The rat brain in stereotaxic coordinates. Academic Press, New York.

Perrett SP, Mauk MD. 1995. Extinction of conditioned eyelid responses requires the anterior lobe of cerebellar cortex. J Neurosci 15: 2074-2080.

Plakke B, Freeman JH, Poremba A. 2007. Metabolic mapping of the rat cerebellum during delay and trace eyeblink conditioning. Neurobiol Learn Mem 88: 11-18.

Poremba A, Gabriel M. 1997. Medial geniculate lesions block amygdalar and cingulothalamic learning-related neuronal acitivty. J Neurosci 17: $8645-8655$.
Poremba A, Gabriel M. 2001. Amygdalar efferents initiate auditory thalamic discriminative training-induced neuronal activity. J Neurosci 21: $270-278$.

Prince JL, Amaral DG. 1981. An autoradiographic study of the projections of the central nucleus of the monkey amygdala. J Neurosci 1: $1242-1259$

Quirk GJ, Repa C, LeDoux JE. 1995. Fear conditioning enhances short-latency auditory responses of lateral amygdala neurons: parallel recordings in the freely behaving rat. Neuron 15: 1029-1039.

Romanski LM, LeDoux JE. 1992. Equipotentiality of thalamo-amygdala and thalamo-cortico-amygdala circuits in auditory fear conditioning. J Neurosci 12: 4501-4509.

Scavio MJ Jr, Gormezano I. 1974. CS intensity effects on rabbit nictitating membrane conditioning, extinction and generalization. Pavlov J Biol Sci 9: 25-34.

Skelton RW. 1988. Bilateral cerebellar lesions disrupt conditioned eyelid responses in unrestrained rats. Behav Neurosci 102: 586-590.

Steinmetz JE, Rosen DJ, Chapman PF, Lavond DG, Thompson RF. 1986. Classical conditioning of the rabbit eyelid response with a mossy-fiber stimulation CS: I. Pontine nuclei and middle cerebellar peduncle stimulation. Behav Neurosci 100: 878-887.

Steinmetz JE, Logan CG, Rosen DJ, Thompson JK, Lavond DG, Thompson RF. 1987. Initial localization of the acoustic conditioned stimulus projection system to the cerebellum essential for classical eyelid conditioning. Proc Natl Acad Sci 84: 3531-3535.

Taub AH, Mintz M. 2010. Amygdala conditioning modulates sensory input to the cerebellum. Neurobiol Learn Mem 94: 521-529.

Tracy JA, Thompson JK, Krupa DJ, Thompson RF. 1998. Evidence of plasticity in the pontocerebellar conditioned stimulus pathway during classical conditioning of the eyeblink response in the rabbit. Behav Neurosci 112: 267-285.

Yeo CH, Hardiman MJ, Glickstein M. 1985. Classical conditioning of the nictitating membrane response of the rabbit. I. Lesions of the cerebellar nuclei. Exp Brain Res 60: 87-98.

Received September 5, 2014; accepted in revised form March 4, 2015. 


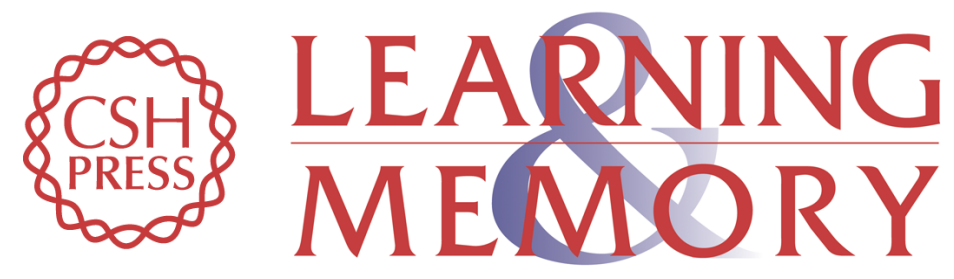

\section{Medial auditory thalamus is necessary for acquisition and retention of eyeblink conditioning to cochlear nucleus stimulation}

Hunter E. Halverson, Amy Poremba and John H. Freeman

Learn. Mem. 2015, 22:

Access the most recent version at doi:10.1101//m.036947.114

\begin{aligned} & \hline References $\begin{array}{l}\text { This article cites } 64 \text { articles, } 26 \text { of which can be accessed free at: } \\ \text { http://learnmem.cshlp.org/content/22/5/258.full.html\#ref-list-1 }\end{array} \\ & \begin{array}{r}\text { Creative } \\ \text { Commons } \\ \text { License }\end{array} \begin{array}{l}\text { This article is distributed exclusively by Cold Spring Harbor Laboratory Press for the } \\ \text { first } 12 \text { months after the full-issue publication date (see } \\ \text { http://learnmem.cshlp.org/site/misc/terms.xhtml). After } 12 \text { months, it is available under } \\ \text { a Creative Commons License (Attribution-NonCommercial } 4.0 \text { International), as } \\ \text { described at http://creativecommons.org/licenses/by-nc/4.0/. }\end{array} \\ & \begin{array}{c}\text { Receive free email alerts when new articles cite this article - sign up in the box at the } \\ \text { top right corner of the article or click here. }\end{array} \\ & \begin{array}{c}\text { Service } \\ \text { terting }\end{array}\end{aligned}$

